\title{
Modelling Dust Emissions from a Source Using Dust Monitoring and Meteorological Data
}

\author{
John Bruce1,2, Jim Smith', Hugh Datson'2, Mike Fowler' \\ ${ }^{1}$ School of Earth and Environmental Sciences, University of Portsmouth, Portsmouth, UK \\ ${ }^{2}$ DustScan Ltd, Oxford, UK \\ Email:JohnB@dustscan.co.uk
}

Received 18 January 2016; accepted 26 February 2016; published 29 February 2016

Copyright (C) 2016 by authors and Scientific Research Publishing Inc.

This work is licensed under the Creative Commons Attribution International License (CC BY). http://creativecommons.org/licenses/by/4.0/

(c) (i) Open Access

\section{Abstract}

This paper describes a study into the development of more robust dust emission factors by means of dust and meteorological monitoring. Emission factors for nuisance dusts in the literature are scarce, with estimates of dust output given for many processes in mass per unit area per year. Temporal variations and the extent and conditions in which maximum concentrations occur can therefore be impossible to predict with any accuracy. This investigation aims to improve predictions by "back calculating" emission levels based on dust monitoring around known dust sources. Nuisance dust and meteorological monitoring has been undertaken at a sand and gravel quarry in the UK for a consecutive period of two years. Sticky pad directional dust monitors were used to collect dust at eight locations at and around the site with meteorological data collected at an electronic weather station within the site. Air quality modelling software (ADMS) was used to test emission factors from the European Environment Agency (EEA) and the US Environmental Protection Agency (EPA) for emissions from mineral workings. Predictions were compared with the dust monitoring data to assess accuracy, with results showing limited poor correspondence $\left(r^{2}<0.3\right)$. Trends showed that emission predictions were poorest in winter; this is likely because most emission calculations are not weather dependent and seasonal fluctuations will occur. Dust emission rate calculations were altered with respect to the dust monitoring data for one monitoring location on the mineral site boundary and the model was run again. Results were then tested at two different locations up to $200 \mathrm{~m}$ from the site boundary, with very positive correlations $\left(\mathrm{r}^{2}>0.89\right)$ and similar maximum concentrations $(<5 \%$ difference). This study has therefore shown that accurate site-specific emission rates can be produced in combination with site boundary sticky pad dust monitoring in order to accurately derive estimations elsewhere.

\section{Keywords}

Dust, Nuisance Dust, Dust Modelling, Sticky Pad, ADMS 


\section{Introduction}

"Dust" as defined in the UK (BS 6069 Part 2 [1]) is described as particulate matter $<75 \mu \mathrm{m}$ in diameter. It can also be differentiated into two size fractions: coarse particles $(>10 \mu \mathrm{m})$ associated with annoyance risks and finer particles linked with risks to human health via inhalation. Coarse particles are undefined and are often referred to as "nuisance dust" or "fugitive dust" and can be chronic, whereby surfaces are soiled from dust settlement, or acute, where problems such as dust clouds cause nuisance. The assessment of nuisance dust in the UK is achieved through criteria developed by the Institute of Air Quality Management (IAQM [2]), National Planning Policy Framework (NPPF [3]) and the Mineral Industry Research Organisation (MIRO [4]).

Nuisance dust from industrial processes and areas of natural dust propagation may have the capacity to cause environmental concern. Dust emissions may be generated by many processes and each must be understood with reference to the volume, content, size and general appearance of the dust.

Nuisance dust modelling is therefore a complex and relatively untested field, compounded by the fact that there are effectively "unlimited" sources and influences. In air quality modelling, for instance, most activities and processes that emit pollutants can be strictly defined and measured to help understand sources and emission rates. For dust emissions however, any process that can cause the entrainment of dust may be deemed an emission. This may include attrition by wind of bare ground or stockpiles, site vehicle movements, or mechanical processes. Individual emission sources are therefore rarely measured, and emission factors in the literature often exist as simple estimates based on site size or materials moved [5]. This paper explains a new technique where dust emissions are modelled with commercial modelling software using existing emission rates from the guidance which are subsequently modified based on dust monitoring data. This will demonstrate that accurate estimations of dust emissions at other sites can be determined from site boundary dust monitoring and that the impact on other, more sensitive, locations can therefore be derived.

\section{Current Methods of Dust Modelling}

Current dust prediction techniques can be split into two broad sub-categories: software modelling and general risk prediction. Risk prediction uses an established method of assessing risk through understanding site characteristics and applying pre-determined risk matrices to give an overall risk value for a site or source. Software modelling uses commercial software based on air dispersion modelling and therefore needs similar inputs. These normally include meteorological data, site data and most importantly emission rate data for each pollutant, from each source. Measured emission rates for individual dust sources however are mostly unavailable and therefore more generalised data are often used which include the estimation of dust emissions on a site-wide basis. Consequently this does not accommodate seasonal fluctuations, site variability or activity and should only be used as an estimate. It may therefore be possible to improve predictions based on comparisons of original emission rates and site-specific measurements. This study will therefore look to examine the potential use of dispersion modelling software in dust impact assessments by generating emission rates based on site specific monitoring data.

\section{Case Study}

For this investigation, commercial air dispersion modelling software was used to test the emission factors available in the literature. Emission factors from the European Environment Agency (EEA) and the US Environmental Protection Agency (EPA) were used to generate dust emission rates for the study site which were used in the dispersion model to predict airborne concentrations for comparison with monitored dust levels.

\subsection{Study Site}

The study site is a small mineral stockyard and quarry in southern England, operating in an area of approximately 3.5 hectares and stockpiling a mixture of aggregates primarily including sands and gravel. An array of eight directional sticky pad dust monitors were located at the site including one upwind of the predominant wind direction, two on the site boundary and a further five downwind to the north and east. Sticky pad samples were collected over consecutive periods of seven to fourteen days; this allowed dust emissions to be monitored in relation to the meteorological patterns and site activities during that period. Dust was continuously monitored for two years and an electronic weather station was established on-site for localised meteorological readings. Site operational data were also provided by the site management. 


\subsection{Model Set Up}

ADMS modelling software [5] was used which requires a number of inputs, including but not limited to meteorological data, terrain and location data (optional), and precise source and emissions data. The software is primarily used for air dispersion modelling, but can also output particle dispersion and deposition. Source data for dust must include particle size grading and density, together with hourly estimations of the emission rate from each individual source.

\subsection{Site Modelling}

Emission factors in the literature can be used to predict dust emissions from sources relevant to mineral workings and stockpile operations. The EEA emissions guidebook [6] states that a mineral site's dust emissions will be directly proportional to the size of the site (16.4 tonnes of total suspended particles (TSP) per hectare per year). The EPA emissions inventory (AP42) [7] states that a proportion of all material transfers (e.g. material taken from or added to stockpiles) will be emitted as dust. Other emission factors can be calculated based on stockpile size and shape; however because the case study site had stockpiles that were constantly excavated, replenished and relocated, these were not used and a different approach was chosen.

Based on observations from visiting the site it was suspected that due to the relatively low activity most of the on-site dust emissions were wind-blown dusts from both the quarry floor and stockpiles, with other dust emissions occurring during the stockpile movements. It was therefore decided that all dust emissions from the site would be modelled as a single area source which covered the floor of the quarry and all of the stockpiles. This prevented the stockpile changes from influencing the results and simplified the modelling process by removing the need for any individual stockpile modelling. Emission factors were used for the site as a whole and for stockpile transfers. Meteorological data input was taken from the on-site weather station.

\subsection{Software Modelling}

An initial problem with comparing the model output with the results from the sticky pad monitors was that the model output was in $\mathrm{mg} \cdot \mathrm{m}^{-3}$, with sticky pad measurements in $\mathrm{mg} \cdot \mathrm{m}^{-2}$ from a cylindrical surface. An additional dust monitor was therefore used to help measure the efficiency of the sticky pad monitors in comparison with dust concentrations in the air passing the monitor. A Wilson and Cook (WAC) dust catcher [7] was installed adjacent to a sticky pad monitor and results were compared with reference to the wind velocities during the collection period. WAC samplers have been shown to have consistently high collection efficiencies relative to different wind velocities ( $90 \%$ efficiency up to $\left.5 \mathrm{~m} \cdot \mathrm{s}^{-1}\right)$ [8] with sticky pad monitors previously shown to have up to $35 \%$ efficiency [9]. Twelve weeks of results were collected that showed that during higher wind velocities the collection efficiency of the sticky pad monitor would drop considerably compared to the WAC sampler, which was assumed to have remained consistent. A model was therefore created that could predict the efficiency of the sticky pad monitor over a given period of time, based on the hourly wind velocities during that interval and the modelled efficiency for each hour.

\section{Results}

\subsection{First Results}

Results from the dust monitoring were converted into mass per unit volume $\left(\mathrm{mg} \cdot \mathrm{m}^{-3}\right)$ using the sticky pad efficiency model and compared with the results from the modelling scenario. Directional dust monitoring results were compared from three key points; one dust monitor at the boundary, Monitor 1 (100 m beyond the boundary) and Monitor 2 (200 m from the boundary). Results only from the arc facing the minerals site were used to limit background dust interference with the trends. Modelling outputs were generated for each monitoring point for direct comparison with dust results; in addition an interpolated gridded output was produced for plotting and to aid the overall understanding of the predicted dust concentrations and spatial variability.

Modelled results (Figure 1) showed limited temporal similarities $\left(r^{2}<0.3\right)$ with the dust monitoring data, although they were generally of the right order; the predicted average concentration at the boundary was 0.093 $\mathrm{mg} \cdot \mathrm{m}^{-3}$ whilst the measured result was $0.054 \mathrm{mg} \cdot \mathrm{m}^{-3}$. The overall trend of the modelled results showed consistent dust levels; this may be because most dust emission calculations do not take into account weather variables other than wind velocity. Large seasonal changes will occur in dust emissions due to temperature and rainfall 


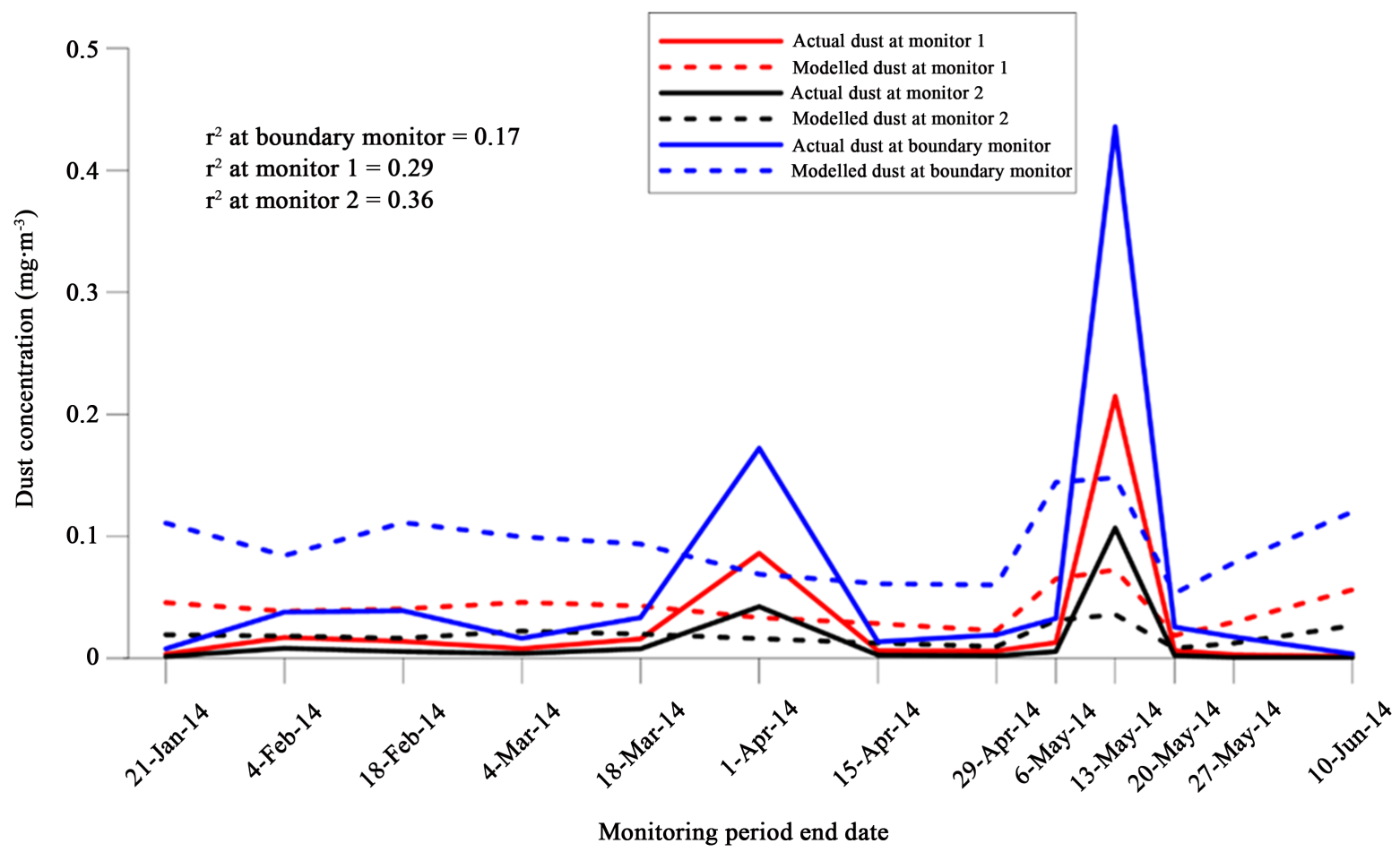

Figure 1. Modelling results vs. dust monitoring results for the study period using published emission factors.

changes that will not be accounted for using basic emission factors [10]. The modelled data did accurately predict the highest period of dust dispersion; this was during the time with the strongest and most consistent high wind velocities.

One other positive was that estimations of the drop-off from the boundary monitor to the monitor furthest away were accurate. This suggested that the model was predicting the dispersion characteristics well but that the source data was not sufficiently accurate.

By re-evaluating the emission rates over the study period in comparison with the dust monitoring results it is possible to adjust the emission rates to equalise results from a single dust monitoring point. The model can then be re-run, with the new "site-specific emission factors" tested against measurements from other dust monitoring locations.

\subsection{New Results}

Changes in dust emissions rates in the model result in proportional changes in the predicted output. For instance, if emissions over one hourly period were modified to be two times higher, and all other variables remained the same, all model outputs such as the predicted concentration or deposition rate would also be twice as high. Because dust emissions were calculated hourly, and averaged per week to compare with the dust monitoring results, each dust monitoring period's emissions were varied by multiplying by the ratio for which they were different. For example, when a dust monitoring period showed values three times as high as those modelled, each individual hourly emission rate during that monitoring period would be multiplied by three. A different correction factor was therefore applied for each dust monitoring period.

The dust monitoring location on the boundary was chosen for "back calculating" as it was thought to have the least influence from other dust sources. New emission rates were therefore calculated for the whole study period and the dispersion model inputs were updated. These new emission rates can therefore be taken as representative for the site as a whole and can be understood by comparison with regard to site operations and meteorological conditions.

The model was run with the new emission rates and predicted concentrations were compared to measured data at two dust monitoring locations in increasing distances from the site boundary. Model results compared very well to dust monitoring results (Figure 2) with an $\mathrm{r}^{2}$ value of 0.98 at Monitor 1 (100 m from the site boundary) 


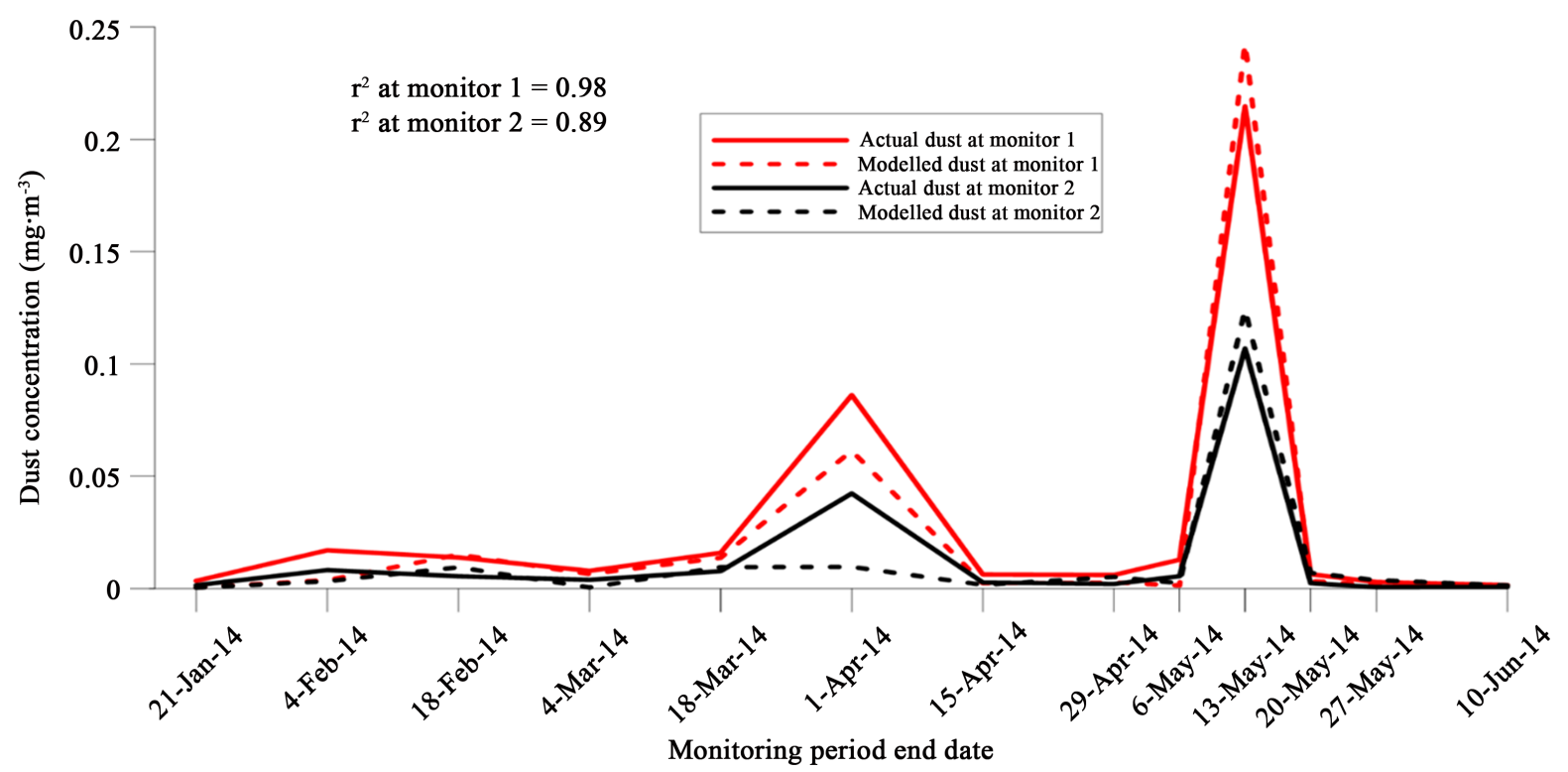

Figure 2. Modelling results vs. dust monitoring results for the study period using "back calculated” emission rates.

and an $\mathrm{r}^{2}$ of 0.89 at Monitor 2 (200 m from the site boundary). Most importantly the highest predicted dust concentration was predicted accurately with a modelled concentration of $0.24 \mathrm{mg} \cdot \mathrm{m}^{-3}$ and a measured concentration of $0.23 \mathrm{mg} \cdot \mathrm{m}^{-3}$. The one monitoring period where the model appeared to under-predict the measured dust levels may be due to other dust sources in the area; there was a recorded increase in farming activity over this time.

\section{Summary}

This study has shown the method of calculating dust emissions from one small site as a single source. An aspect therefore not examined in this study, but with which work is ongoing, is the source apportionment of dusts being predicted from several separate dust sources. This would allow for the attribution of dusts from specific sources and processes and may help assess the importance of different dust components.

The improvement in model comparisons after the back calculation of emission rates indicates that when emissions are accurately entered into models, subsequent dust dispersion may be predicted with much greater accuracy. This can be achieved using a boundary dust monitor to derive emission rates with other, more sensitive, locations extrapolated via modelling. The key to using air dispersion models to their full capacity for dust dispersion is therefore the creation and measurement of a more thorough and adjustable emission factor database for specific dust sources. This should include variables with major influences such as temperature and rainfall that currently are not accommodated in many of the EEA or EPA emissions factors. This would ultimately allow sites in the planning stages, or those without dust monitoring, to be more accurately modelled instead of estimating emissions based on general characteristics. This method also demonstrates that emissions for existing sites can be understood by calculating "bespoke" dust emission rates based and tested on site specific dust monitoring. Recommendations for future improvements of the methodology include the understanding of specific dust sources through characterisation, and the need to analyse how the new emission rates can be related to paratmers such as rainfall and temperature. Further work currently underway also includes trials at other site types such as construction and demolition.

Overall this study has shown that published emission rates may not be suitable for predicting weekly variations or maximum concentrations of dust emissions at mineral sites where several activities may take place concurrently. The collection of dust monitoring data is key to understanding why and how emissions will vary and new emission rates can be easily "back calculated" for use in air dispersion models. Ultimately this would allow for a new database to be developed to include emission rates for multiple site types, including but not limited to: construction and demolition sites, waste and recycling sites, and general industrial sites. 


\section{Acknowledgements}

We would like to thank Innovate UK (formerly the Technology Strategy Board) for helping fund the Knowledge Transfer Partnership during which much of this work took place. We are also grateful towards Professor Geoffrey Walton for his continued support and assistance that made this work possible.

\section{References}

[1] BSI (1994) BS 6069 Part 2. Characterisation of Air Quality, Part 2, Glossary. British Standards Institution, London.

[2] Institute of Air Quality Management (IAQM) (2012) Guidance on Air Quality Monitoring in the Vicinity of Demolition and Construction Sites. Institute of Environmental Sciences, London.

[3] Department for Communities and Local Government (DCLG) (2012) National Planning Policy Framework (NPFF). HMSO, London.

[4] Minerals Industry Research Organisation (2011) Good Practice Guide: Control and Measurement of Nuisance Dust and PM10 from the Extractive Industries. AEA Technology, Oxford.

[5] Cambridge Environmental Research Consultants (CERC) (2015) ADMS 5. http://www.cerc.co.uk/environmental-software/ADMS-model.html

[6] European Environment Agency (2013) EMEP/EEA Air Pollutant Emission Inventory Guidebook. EEA, Denmark.

[7] Environmental Protection Agency (EPA) (2006) AP42: 13.2.4 Aggregate Handling and Storage Piles. EPA, Washington DC.

[8] Goosens, D. and Offer, Z.Y. (2000) Wind Tunnel and Field Calibration of Six Aeolian Dust Samplers. Atmospheric Environment, 34, 1043-1057. http://dx.doi.org/10.1016/S1352-2310(99)00376-3

[9] Datson, H. (2010) The Development of a New Protocol for Directional Dust Monitoring. PhD Thesis, University of Leeds, UK.

[10] Bruce, J., Datson, H., Smith, J. and Fowler, M. (2015) Characterisation and Modelling of Dust in a Semi-Arid Construction Environment. In: O’Sullivan, G. and Megson, D., Eds., Environemntal Forensics: Proceedings of the 2014 INEF Conference, Special Publication, The Royal Society of Chemistry, London. 